\title{
Upward transtentorial herniation following frontoorbital advancement for syndromic craniosynostosis: case report
}

\author{
Vivek Mehta, MD, ${ }^{1}$ Joshua Bakhsheshian, MS, ${ }^{2}$ Amir H. Dorafshar, $\mathrm{MBChB},{ }^{3}$ and \\ Edward S. Ahn, MD4 \\ 'Department of Neurosurgery, USC Keck School of Medicine, Los Angeles, California; ${ }^{2}$ Chicago Medical School at Rosalind \\ Franklin University of Medicine \& Science, North Chicago, Illinois; and ${ }^{3}$ Department of Plastic and Reconstructive Surgery and \\ ${ }^{4}$ Division of Pediatric Neurosurgery, Johns Hopkins University School of Medicine, Baltimore, Maryland
}

\begin{abstract}
The authors describe the case of a boy with Muenke syndrome, an autosomal dominant disorder associated with craniosynostosis. The family history was significant for syndromic craniosynostosis in the patient's maternal grandmother, who died in adulthood after a craniofacial reconstruction. The patient, her grandson, underwent craniofacial reconstruction surgery at the age of 9 months and developed upward transtentorial herniation. Imaging findings revealed remote cerebellar hemorrhage after a large quantity of supratentorial CSF was drained during postoperative Day 1 . The clinical course was further complicated by cerebral sinus thrombosis, which was diagnosed after a fourth surgical procedure. Upward transtentorial herniation can occur when a significant increase in intracranial pressure in the posterior fossa causes displacement of the central lobule and superior surfaces of the cerebellum upward through the incisura tentorii. This is a rare but well-documented phenomenon that commonly occurs in the setting of an expansive posterior fossa lesion or excessive supratentorial CSF loss. To help clinicians recognize and prevent this rare but potentially fatal complication, the authors review the postulated mechanisms by which this process may occur.
\end{abstract}

http://thejns.org/doi/abs/10.3171/2015.2.FOCUS151

KEY WORDS remote intracranial hemorrhage; herniation; intracranial pressure

$\mathrm{M}$ UENKE syndrome is an autosomal dominant inherited disorder defined by the presence of a specific FGFR3 mutation; it accounts for $25 \%-30 \%$ of all genetic causes of craniosynostosis. ${ }^{1}$ Premature fusion of calvarial sutures can lead to increased intracranial pressure (ICP), progression of cranial deformity, and other complications. ${ }^{2}$ Therefore, patients with Muenke syndrome and craniosynostosis often undergo frontoorbital advancement and cranial vault remodeling. ${ }^{1}$ Early surgical reconstruction for craniosynostosis can reduce the risk of postoperative complications, which include increased ICP and the need for secondary or tertiary revision procedures. A national patient database demonstrated that the overall complication rate following surgical repair of craniosynostosis is $10.1 \%$, with hemorrhage being the most frequent complication. ${ }^{2}$ The complication rates are even higher in patients undergoing reoperation.

Remote cerebellar hemorrhage $(\mathrm{RCH})$ is a rare complication of supratentorial surgery and, to our best knowl- edge, has not previously been reported as a complication of craniosynostosis repair. While RCH is often self-limiting, ${ }^{3,7}$ early recognition and treatment can prevent further dire complications. ${ }^{5}$ Severe complications may involve neurological deterioration due to the resulting cerebellar edema, marked hydrocephalus, and brainstem compression by upward transtentorial herniation. ${ }^{6}$ We describe a case in which excessive loss of supratentorial CSF and subsequent $\mathrm{RCH}$ following craniosynostosis repair led to upward transtentorial herniation. We also review the postulated mechanisms by which this may occur in order to help future clinicians recognize and prevent this rare complication.

\section{Case Report}

History and Examination

This patient presented shortly after birth because of concern for craniosynostosis associated with Muenke syndrome. The diagnosis of Muenke syndrome had been

ABBREVIATIONS ICP = intracranial pressure; $\mathrm{RCH}=$ remote cerebellar hemorrhage .

SUBMITTED January 1, 2015. ACCEPTED February 25, 2015.

INCLUDE WHEN CITING DOI: 10.3171/2015.2.FOCUS151.

DISCLOSURE The authors report no conflict of interest concerning the materials or methods used in this study or the findings specified in this paper. 
established prenatally by amniocentesis, which was performed because of a strong family history. The child's mother and maternal grandmother had been diagnosed with Crouzon's disease, but this was more likely to have been unrecognized Muenke syndrome. The patient's grandmother underwent strip coronal synostosis release as an infant and then at age 29 underwent a frontal bone remodeling/maxillary reconstruction procedure. Her postoperative course was complicated; on postoperative Day 2 neurological decline, severe hyponatremia, seizures, and cardiopulmonary arrest developed. She was resuscitated, but a postresuscitation CT scan demonstrated evidence of cerebral edema and, eventually, uncal herniation. She subsequently progressed to brain death.

At 1 month of age, our patient's anterior and posterior fontanels were open and soft. There was bridging across both coronal sutures resulting in synostotic brachycephaly with restriction in the supraorbital region. By 7 months, his head circumference was above the 95th percentile for his age group, and he had noticeable craniofacial abnormalities, including frontal bossing, turricephaly, brachycephaly, and an impalpable anterior fontanel. The bilateral coronal sutures were fused with advancement of the sphenoid wings bilaterally (Fig. 1). Bifrontal craniotomy with frontoorbital advancement was planned for the following month.

\section{Operations and Complications}

A bifrontal craniotomy with bilateral parietal craniotomies for frontoorbital advancement was performed at 8 months. During the bifrontal craniotomy and orbital bar removal, 2 durotomies occurred and were repaired primarily. The patient tolerated the procedure well. Twelve hours after surgery, the subgaleal Hemovac drain output was $365 \mathrm{ml}$, and a CSF leak through the durotomies was suspected. The patient developed a precipitous decline in mental status, with bradycardia and hypertension. A head CT scan demonstrated findings of subarachnoid hemorrhage in the cerebellar folia with moderate surrounding vasogenic edema and effacement of the fourth ventricle (Fig. 2). There was also a small amount of subdural hematoma layering along the tentorium. He was emergently intubated, given mannitol, and resuscitated and was then taken to the operating room for a posterior fossa decompression. A wide suboccipital craniectomy was performed. The dura was found to be pulsating spontaneously without evidence of tension. Ultrasound visualization demonstrated an open fourth ventricle and visible tips of the cerebellar tonsils. The patient's vital signs were stable throughout the operation. After extubation the patient demonstrated stridor that was responsive to racemic epinephrine, and he was weaned to room air a day later. He remained stable for 3 days following the operation and then developed several episodes of bradycardia. A head CT scan revealed progression of the hydrocephalus due to obstruction from the posterior fossa edema (Fig. 3). The patient was emergently taken to the operating room for insertion of an intraventricular catheter. He was extubated and stridor was noted again. He demonstrated bradycardia that was unresponsive to mechanical ventilation and infusion of hypertonic saline. Two days later, he developed a dysconjugate gaze

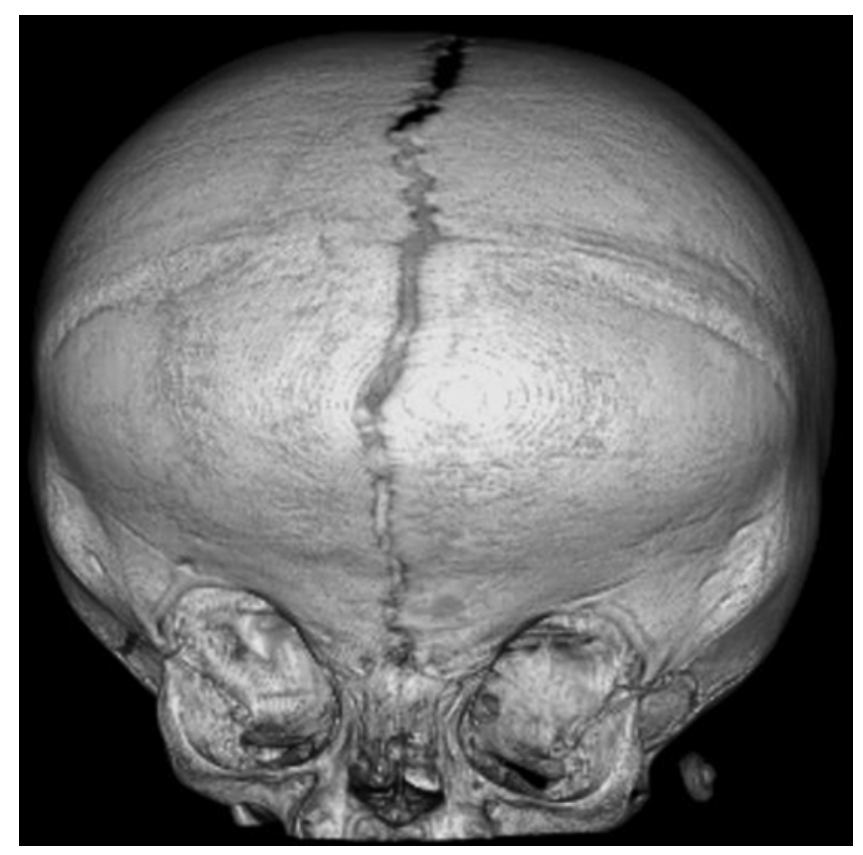

FIG. 1. Preoperative CT reconstruction.

and had persistent ICP elevated over $25 \mathrm{~mm} \mathrm{Hg}$. Head CT demonstrated persistent cerebellar edema with a small fourth ventricle and compression at the cervicomedullary junction (Fig. 4 left). The patient was taken to the operating room for a revision of the suboccipital craniectomy and a duraplasty to further decompress the brainstem. Following this fourth procedure, he was noted to have an acute increase in ICP with sustained pressure recordings above $27 \mathrm{~mm} \mathrm{Hg}$. An MR venogram confirmed the presence of a venous sinus thrombosis in the left transverse and sigmoid sinuses (Fig. 4 right). Anticoagulation with unfractionated heparin was initiated, followed by low-molecular-weight heparin. The rest of his hospital stay was prolonged but uncomplicated. The patient was discharged with mild esotropia that did not require treatment. He completed anticoagulation therapy without any further sequelae of the venous sinus thrombosis.

At age 2 years, he presented with bacterial meningitis and generalized tonic-clonic seizures, which were controlled with antiepileptic medication. He remained seizure free for 3 years and medications were weaned. There were gross motor and speech delays. At age 5, he is meeting motor milestones. Delays in speech development persist, such as stringing together of 2- to 3-word phrases. He underwent scar revision and filling of temporal hollowing with fat grafts without complication.

\section{Discussion}

Remote cerebellar hemorrhage $(\mathrm{RCH})$ is a rare complication occurring in $0.12 \%$ to $3.5 \%$ of supratentorial surgeries. ${ }^{20}$ It has been reported to occur with craniotomies for removal of supratentorial cerebral content or CSF. $\mathrm{RCH}$ has a typical appearance on CT imaging, with curvilinear collections of blood that parallel the superior folia and horizontal fissure and extend toward the tentorium. This appearance is referred to as the zebra sign (Fig. 2). 

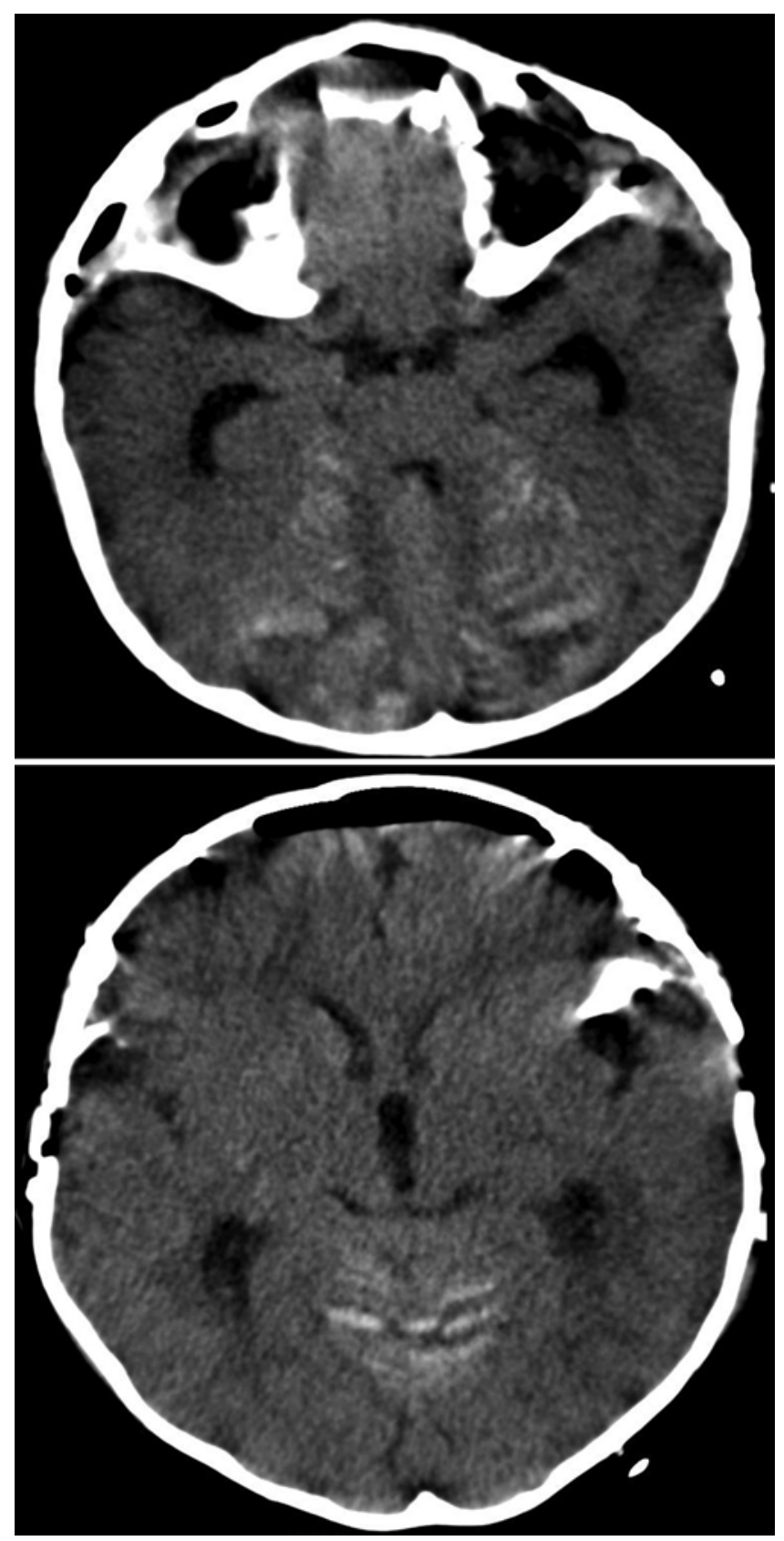

FIG. 2. Upper: Representative axial CT scan immediately following neurological decline. Lower: Curvilinear collections of blood that parallel the superior folia and fissure toward the tentorium, which is referred to as the zebra sign.

While $\mathrm{RCH}$ can be observed in postoperative images of asymptomatic patients and can have a benign course, ${ }^{3,7}$ it can also present with cerebellar and brainstem dysfunction. ${ }^{5} \mathrm{RCH}$ can occur after craniosynostosis repair and may increase the risk for upward supratentorial herniation.

The mechanism of $\mathrm{RCH}$ from supratentorial procedures is not completely understood. However, numerous theories have been proposed (Fig. 5). König et al. were the first to suggest a reciprocal relationship between the removal of supratentorial contents, such as tumors or CSF, and a resulting rise in venous pressure eventually leading

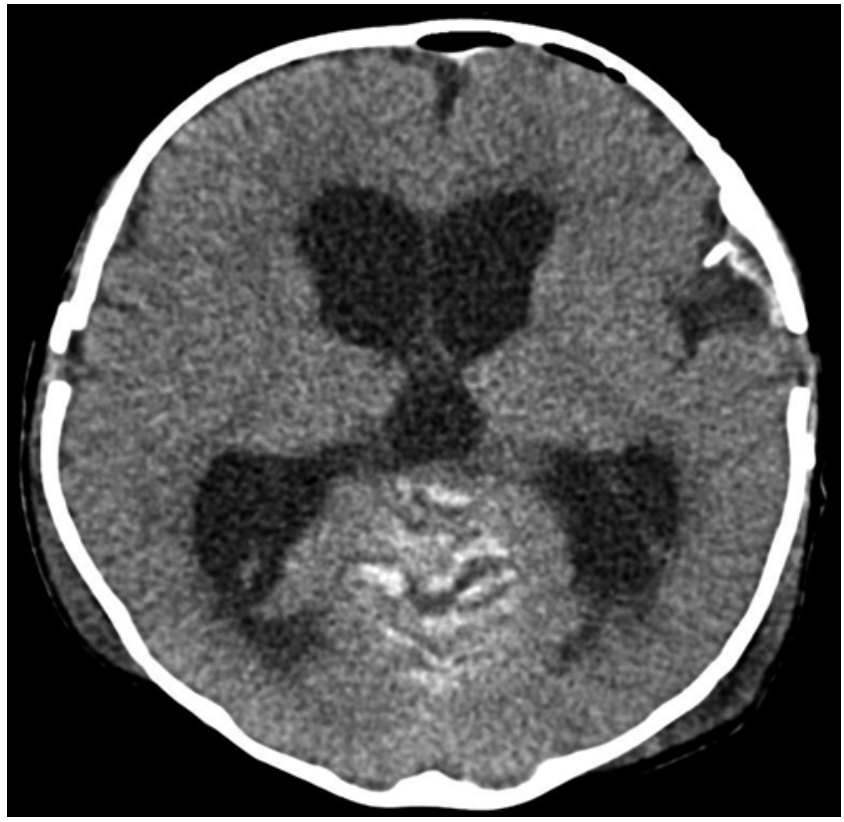

FIG. 3. Axial CT scan showing progression of the hydrocephalus.

to hemorrhage. ${ }^{15}$ Later, Yoshida et al. suggested that excess CSF drainage causes stretching of the superior vermian vein and its tributaries, which leads to hemorrhage. ${ }^{21}$ Friedman et al. agreed with the downward displacement theory but speculated that cerebellar sag and venous occlusion lead to hemorrhagic venous infarction. ${ }^{9}$ Honegger et al. disagreed with previous theories and suggested that postoperative suction of excess supratentorial CSF results in a negative pressure gradient that damages the venous channels and capillary bed of the cerebellum. ${ }^{11}$ Honegger et al. also noted that deliberate obliteration of supracerebellar veins during infratentorial supracerebellar surgery does not produce cerebellar hemorrhage, thereby discrediting the belief that cerebellar veins are the culprit behind $\mathrm{RCH}$.

Although there is still a fair amount of debate over the exact pathomechanism of $\mathrm{RCH}$, a general consensus exists regarding certain risk factors, including arterial hypertension, intraoperative opening of the cisterns, excessive amounts of acute postoperative CSF drainage, large
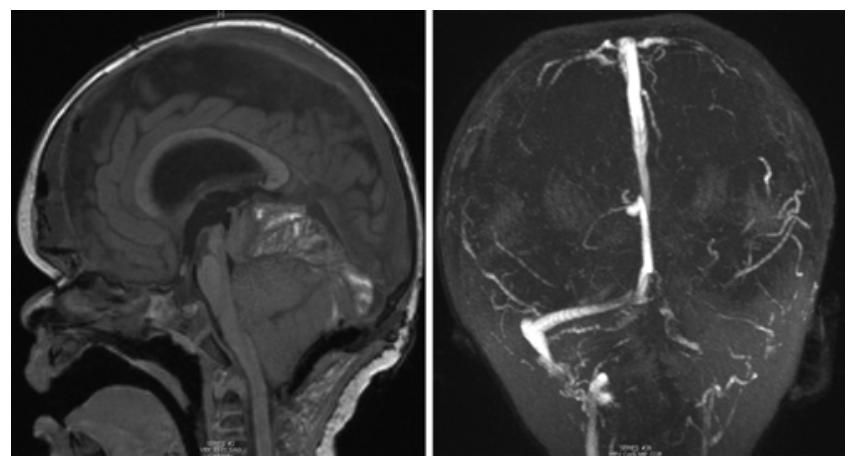

FIG. 4. Left: Sagittal T1-weighted MR image demonstrating superior cerebellar hemorrhages and brainstem compression. Right: MR venogram confirming the presence of a venous sinus thrombosis in the left transverse and sigmoid sinuses. 


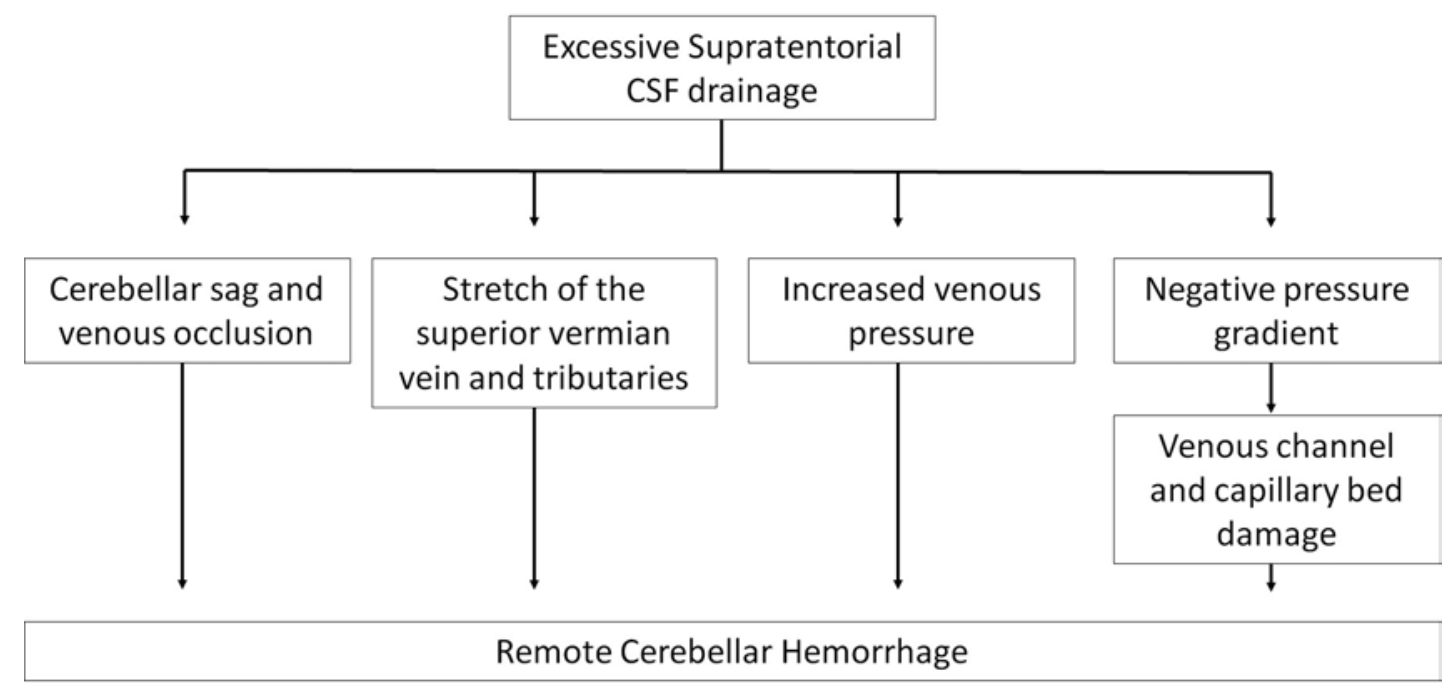

FIG. 5. Postulated mechanisms for upward transtentorial hernation.

resection cavities, and drain insertion. While the focus of our report is on supratentorial procedures, it should be noted that acute loss of CSF after spinal procedures may also cause the same subarachnoid bleeding pattern in the cerebellum. ${ }^{13}$ In our case, $\mathrm{RCH}$ was observed after a large amount of supratentorial CSF fluid was drained.

Postoperative CT imaging can help diagnose and assess the natural evolution of the hemorrhage. ${ }^{3}$ The zebra sign is a curvilinear collection of blood that parallels the superior folia and horizontal fissure and extends toward the tentorium after spinal or supratentorial procedures that lead to CSF loss. ${ }^{5}$ Postoperative imaging can further demonstrate early signs of upward herniation, such as compression and slight posterior flattening of the quadrigeminal plate cistern. Severe RCH is characterized by herniation of the vermis, which results in plugging of the incisura, complete effacement of the cistern, and flattening of the posterior third ventricle. Obstructive hydrocephalus may also occur with moderate or severe herniation.

Mortality rates due to spontaneous cerebellar hemorrhages can range from $20 \%$ to $75 \%$. However, the rate of $\mathrm{RCH}$ following supratentorial craniotomy is lower, ranging from $7.8 \%$ to $14.8 \% .^{4,16}$ Many studies have used management approaches similar to those used for spontaneous cerebellar hematoma, even though the natural history of the two entities is different. ${ }^{14}$

The literature on the management of $\mathrm{RCH}$ is limited to retrospective clinical studies of small cohorts, mainly adult patients. $\mathrm{RCH}$ after a supratentorial craniotomy can be treated conservatively or decompressed using a ventricular drain or craniectomy with good clinical outcomes., ${ }^{40,12,16,19}$ With excessive loss of CSF and RCH observed on CT imaging, drains should be discontinued to prevent exacerbation of the $\mathrm{RCH} .{ }^{4}$ Other authors have recommended against the use of suction drainage ${ }^{11,21}$ and intraoperative mannitol for procedures at higher risk of $\mathrm{RCH}$ (hemorrhage severity and patient age). ${ }^{11}$

In this report, a postoperative drain to bulb suction was placed due to the large craniotomy and bleeding. We would not recommend wall suction with or without dural tear. Thumbprint suction can be applied with a JacksonPratt drain, or the drain may be set for minimal negative pressure to avoid draining a large volume of CSF. A direct causal relationship between the use of postoperative drainage and $\mathrm{RCH}$ has not been established. ${ }^{9}$ The difficulties in establishing a direct relationship may be due to the rarity of this complication. In a retrospective cohort analysis, Huang et al. were able to demonstrate a significant correlation between postoperative epidural drainage amount $(221 \mathrm{ml}$, as opposed to $100 \mathrm{ml}$ in the control patients) and $\mathrm{RCH}$ at 4 hours postoperatively. ${ }^{12}$ Drain output should be cautiously observed for volume and quality to avoid excessive CSF drainage, especially in the immediate postoperative period. If drainage is due to scalp bleeding, removing the drain can lead to compression by an epidural hematoma, which may cause downward herniation.

Redistribution of CSF between the spinal and supratentorial compartments is expected after drain removal. If $\mathrm{RCH}$ is suspected, infusion of Ringer's solution to replace lost CSF can be beneficial if obstructive hydrocephalus has not developed. ${ }^{4}$ The development of obstructive hydrocephalus would require a ventricular drainage system. ${ }^{4}$ Larger hemorrhages or signs of herniation may indicate the need for immediate surgical decompression. The decision to intervene is ultimately based on the patient's clinical course and findings on CT imaging.

Cerebral venous sinus thrombosis was diagnosed in the patient following the fourth operative procedure. Thrombosis of the cerebral sinuses can result in increased vascular pressure, parenchymal hemorrhage, and decrease CSF absorption, causing an increased ICP and increased risk for herniation. ${ }^{17}$ It is possible that the venous sinus thrombosis was a missed event, since we did not have a prior venogram that would have established the timing of the thrombosis during the sequence of events. Acute control of elevated ICP must be achieved in the safest way possible. Controlling the high ICP in the present case was difficult. Hyperosmolar agents should be administered to patients at risk for herniation, ${ }^{8}$ but the use of mannitol may have precipitated the sinus thrombosis due to its dehydrat- 
ing mechanism. Alternatively, hypertonic saline can be used if there is a concern for a hypercoaguable state or the precipitation of a sinus thrombosis.

It is difficult to confirm our hypothesis without data regarding the change in ICP following CSF drainage; however, it correlates with previous published reports. The pathophysiology may also be different in the young pediatric population. Typically the cranial cavity is not rigid in infants as the cranial sutures can continue to expand. In cases of syndromic craniosynostosis, an increase in intracranial volume will more likely result in a larger increase in ICP than in an infant with open sutures. RCH is extremely rare in children, ${ }^{18}$ and our case report may describe the youngest patient to incur RCH after a supratentorial craniotomy procedure.

\section{Conclusions}

Frontoorbital advancement for syndromic craniosynostosis led to an acute change and imbalance in the volume of all intracranial contents (blood, CSF, cranial vault capacity) in our patient. This imbalance caused an acute neurological deterioration. The loss of CSF from the supratentorial compartment ultimately caused upward herniation through a variety of proposed mechanisms. We identified $\mathrm{RCH}$ and venous sinus thrombosis during the clinical decline of our patient, and these may have caused or increased the risk for upward herniation. We recommend an increased awareness of these rare clinical complications in treating pediatric patients with syndromic craniosynostosis and loss of high volumes of CSF.

\section{References}

1. Agochukwu NB, Doherty ES, Muenke M: Muenke Syndrome, in Pagon RA, Adam MP, Ardinger HH, et al (eds): GeneReviews. Seattle: University of Washington, 2014

2. Allareddy V: Prevalence and impact of complications on hospitalization outcomes following surgical repair for craniosynostosis. J Oral Maxillofac Surg 72:2522-2530, 2014

3. Amini A, Osborn AG, McCall TD, Couldwell WT: Remote cerebellar hemorrhage. AJNR Am J Neuroradiol 27:387390, 2006

4. Brockmann MA, Groden C: Remote cerebellar hemorrhage: a review. Cerebellum 5:64-68, 2006

5. Brockmann MA, Nowak G, Reusche E, Russlies M, Petersen D: Zebra sign: cerebellar bleeding pattern characteristic of cerebrospinal fluid loss. Case report. J Neurosurg 102:11591162,2005

6. Chen HJ, Lee TC, Wei CP: Treatment of cerebellar infarction by decompressive suboccipital craniectomy. Stroke 23:957961,1992

7. Dincer A, Özcan Ü, Kaya D, Usseli MI, Erzen C, Pamir MN: Asymptomatic remote cerebellar hemorrhage: CT and MRI findings. Cerebellum 11:880-886, 2012

8. Ferro JM, Canhão P: Cerebral venous sinus thrombosis: update on diagnosis and management. Curr Cardiol Rep 16:523, 2014

9. Friedman JA, Piepgras DG, Duke DA, McClelland RL,
Bechtle PS, Maher CO, et al: Remote cerebellar hemorrhage after supratentorial surgery. Neurosurgery 49:1327-1340, 2001

10. Ha SH, Kim EM, Ju HM, Lee WK, Min KT: Remote cerebellar hemorrhage after unruptured cerebral aneurysm surgery: two cases report. Korean J Anesthesiol 67:213-216, 2014

11. Honegger J, Zentner J, Spreer J, Carmona H, Schulze-Bonhage A: Cerebellar hemorrhage arising postoperatively as a complication of supratentorial surgery: a retrospective study. J Neurosurg 96:248-254, 2002

12. Huang CY, Lee PH, Lin SH, Chuang MT, Sun YT, Hung YC, et al: Remote cerebellar hemorrhage following supratentorial craniotomy. Neurol Res 34:422-429, 2012

13. Karaeminogullari O, Atalay B, Sahin O, Ozalay M, Demirors H, Tuncay C, et al: Remote cerebellar hemorrhage after a spinal surgery complicated by dural tear: case report and literature review. Neurosurgery (1 Suppl) 57:E215, 2005

14. Kirollos RW, Tyagi AK, Ross SA, van Hille PT, Marks PV: Management of spontaneous cerebellar hematomas: a prospective treatment protocol. Neurosurgery 49:1378-1387, 2001

15. König A, Laas R, Herrmann HD: Cerebellar haemorrhage as a complication after supratentorial craniotomy. Acta Neurochir (Wien) 88:104-108, 1987

16. Park JS, Hwang JH, Park J, Hamm IS, Park YM: Remote cerebellar hemorrhage complicated after supratentorial surgery: retrospective study with review of articles. J Korean Neurosurg Soc 46:136-143, 2009

17. Piazza G: Cerebral venous thrombosis. Circulation 125:1704-1709, 2012

18. Sasani M, Ozer AF, Oktenoglu T, Karaarslan E, Sasani H, Kaner T: Remote cerebellar hemorrhage following resection of a supratentorial tumor: a case report. Cases J 2:7299, 2009

19. Smith R, Kebriaei M, Gard A, Thorell W, Surdell D: Remote cerebellar hemorrhage following supratentorial cerebrovascular surgery. J Clin Neurosci 21:673-676, 2014

20. Thangasamy SJ, Vagal A, Radhakrishnan R: Remote cerebellar hemorrhage as an unusual complication of supratentorial surgery. A case report and literature review. Neuroradiol J 24:779-782, 2011

21. Yoshida S, Yonekawa Y, Yamashita K, Ihara I, Morooka Y: Cerebellar hemorrhage after supratentorial craniotomy-report of three cases. Neurol Med Chir (Tokyo) 30:738-743, 1990

\section{Author Contributions}

Conception and design: Ahn, Mehta. Acquisition of data: Ahn, Mehta, Dorafshar. Analysis and interpretation of data: all authors. Drafting the article: Ahn, Mehta, Bakhsheshian. Critically revising the article: all authors. Reviewed submitted version of manuscript: all authors. Approved the final version of the manuscript on behalf of all authors: Ahn. Administrative/technical/material support: Bakhsheshian. Study supervision: Ahn, Mehta.

\section{Correspondence}

Edward S. Ahn, Division of Pediatric Neurosurgery, Johns Hopkins University School of Medicine, 600 N. Wolfe St., Phipps 560, Baltimore, MD 21287. email: eahn4@jhmi.edu. 